\title{
A Survey of Clarithromycin Monotherapy for Patients with MAC Lung Disease in Japan
}

\section{Tomohide Iwao*}

Nara Institute of Science and Technology, Institute for Research Initiatives, Ikoma City, Japan

\begin{abstract}
Background: The number of patients with nontuberculous mycobacteriosis (NTM) has increased exponentially in recent years. In Japan, approximately $88.8 \%$ of patients with NTM suffer from Mycobacterium avium-intracellulare complex (MAC) Lung Disease. Incidence of MAC Lung Disease is increasing owing to a rapid increase in nontuberculous mycobacterial infections.

Although basic treatment for MAC Lung Disease is chemotherapy, clarithromycin monotherapy is contraindicated by specialists owing to its high potential to induce drug-resistant bacterial strains in patients with MAC Lung Disease. However, it is unclear how much such treatment cases exist in clinical practical. This is because no longterm investigation has been carried out.
\end{abstract}

Materials and Methods: This study investigated treatment with these medications from 2005 to 2017, by studying 1107 patients with MAC Lung Disease based on data in patient insurance claims.

Results: Results showed that approximately 10.3\% (114 cases) were prescribed long-term CAM monotherapy for three months.

Conclusion: Generally, administration of antibiotics over a long period of time is potentially detrimental to some patients. Better awareness of the types of treatments and their potential negative effects will be beneficial to clinical practitioners. In the immediate future, it seems to be effective to develop an educational system on single agent administration of antimicrobial agents.

Keywords: Clarithromycin monotherapy; MAC lung disease; Nontuberculous mycobacteriosis

Key Points: CAM monotherapy for patients with Mycobacterium avium-intracellulare complex (MAC) Lung Disease is not recommended.

The reason is that there is a possibility of inducing resistant bacteria.

However, it is unclear how much such treatment cases exist in clinical practical.

This study investigated treatment with these medications using the database of insurance claims from 2005 to 2017 and identified 1107 patients with MAC Lung Disease.

Results showed that approximately $10.3 \%$ (114 cases) were prescribed long-term CAM monotherapy for three months or longer.

\section{INTRODUCTION}

Nontuberculous mycobacteriosis (NTM) is becoming a greater public health problem associated with an increasing financial burden, particularly in elderly patients [1]. This trend is particularly prominent in Japan. A 2014 nationwide survey in Japan found that 14.7 out of every 100,000 people are infected with NTM, approximately 2.6 times higher than the number reported in the same nationwide survey conducted in 20072. Furthermore, Mycobacterium avium-intracellulare complex (MAC) Lung Disease

Correspondence to: Tomohide Iwao, Nara Institute of Science and Technology, Institute for Research Initiatives, Takayama Town 8916-5 in Ikoma City, Japan, Telephone: +81-743-72-6065, E-mail: iwao@is.naist.jpu

Received: January 31, 2019; Accepted: February 28, 2019; Published: March 07, 2019

Citation: Iwao T (2019) A Survey of Clarithromycin Monotherapy for Patients with MAC Lung Disease in Japan. Adv Pharmacoepidemiol Drug Saf 8:224.

Copyright: (c) 2019 Iwao T. This is an open-access article distributed under the terms of the Creative Commons Attribution License, which permits unrestricted use, distribution, and reproduction in any medium, provided the original author and source are credited. 
accounts for $88.8 \%$ of NTM, M. kansasii accounts for $4.3 \%$ and M. abscessus disease accounts for 3.3\% [2]. MAC Lung Disease is increasing among middle-aged and elderly women and is difficult to cure completely due to moderate effectiveness of antibiotics. This study will focus mainly on MAC Lung Disease which is the major factor in the rapid increase of NTM.

MAC Lung Disease is typically treated by either surgical removal of the infected area or chemotherapy using antibiotics. Although surgical treatments are performed in some cases, chemotherapy with antibiotics is the most common approach. A triple-agent therapy consisting of clarithromycin (CAM), ethambutol (EB) and rifampicin is recommended by specialists as the standard treatment. Dual-agent therapy with streptomycin and kanamycin may also be recommended based on the needs of the patients [3]. ATS guidelines state that an antibiotics regimen for a period of one year or longer from bacilli negative conversion is necessary because MAC Lung Disease with mycobacteriosis is unusually difficult to treat due to moderate effectiveness of antibiotics [4]. However, while clear evidence for a specific treatment period has not been established, guidelines from the UK suggest chemotherapy for MAC Lung disease should continue for a minimum of 12 months after culture conversion [5] and Japan has also reported that treatment periods for longer than what is suggested by ATS have led to better outcome [6]. Furthermore, in successful treatments, bacilli negative conversion generally occurred within six months from the start of antibiotic administration [7].

If the chemotherapy regimen for MAC Lung Disease is interrupted, the effectiveness of the treatment is weakened when treatment is restarted, resulting in poor outcome [8]. In addition, the recommended regimens for treatment of MAC Lung Disease are associated with numerous side effects owing to the use of multiple drugs and long duration of treatment [9]. Therefore, it is important to complete appropriate chemotherapy regimen because there is major risk of inducing multi-agent drug resistant bacterial strains by administering CAM monotherapy. Because it is reported that longterm CAM monotherapy induced CAM-resistant bacterial strains at an early stage (between the $2^{\text {nd }}$ and $5^{\text {th }}$ months) [4]. However, it is unclear what percentages of patients receive CAM monotherapy in clinical practice because long-term, multi-year studies on drug prescriptions for MAC Lung Disease have not been performed. This study aims to quantify the types of treatment strategies used in clinical practice by conducting retrospective cohort research using data from insurance claims. The purpose of an insurance claim is to request the cost of medical care from the medical insurer. Claims consist of a bill from medical institutions or insurance pharmacies with details of medical examinations performed on the patient and prescribed medications issued on a monthly basis in Japan.

\section{MATERIALS AND METHODS}

\section{Data source}

This study used data from insurance claims taken from the Japan Medical Data Center (JMDC). The JMDC compiles information from insurance claims made by patients insured through societymanaged health insurance plans. As of June 2018, the JMDC had completely anonymized the insurance claim information of 5.4 million insured patients aged 0 to 74 years old [10].

\section{Extraction conditions for the insured}

In Japan, the diagnostic criteria for pulmonary NTM are determined by clinical criteria from thoracic imaging and bacteriological analysis, such as sputum test, obtained during examinations [3]. In addition, the ICD-10 code system consolidates NTM into the following codes: A310 (Pulmonary mycobacterial infection), A311 (Cutaneous mycobacterial infection), A318 (Other bacterial diseases) and A319 (Mycobacterial infection, unspecified). Based on this, we established the extraction conditions for insurance claim data from the JMDC; all insured patients who were assigned any of the ICD-10 codes for NTM-A310, A311, A318, or A319-more than once within an observation period of 13 years, from January 2005 to December 2017 were included.

\section{Extraction data}

We obtained data in CSV format (data size $5 \mathrm{~GB}$ ) on patient background (patient ID, age, gender), all injury and disease history and medication history for 16,247 patients that matched the extraction criteria. In addition to this data, we received only background data for a population of 5,478,478 from January 2005 to December 2017.

\section{Patient eligibility criteria and data analysis}

Patient eligibility criteria: First, we selected patients assigned the ICD-10 code A310 because this study is concerned with patients with pulmonary diseases (Eligibility Criterion). To be included in the study, patients must have been prescribed CAM (Eligibility Criterion). Patients with MAC Lung Disease must have been prescribed CAM at least twice to account for the possibility that patients prescribed CAM only once could have had a different ailment such as a cold.

We also set an exclusion criterion to remove patients with diseases that were difficult to distinguish from MAC Lung Disease at the time of diagnosis. Specifically, we excluded patients who were prescribed pyrazinamide, isoniazid or imipenem, which are firstline treatments for pulmonary tuberculosis, M. kansasii disease and M. abscessus disease.

Data analysis: We converted the CSV files obtained from the JMDC into a database, added attributes to the ID for the insured individuals such as age, medication prescribed, dose (mg) prescribed and date of prescription, then created a database for ID of each insured individual. Next, we evaluated patient eligibility criteria. Finally, we determined the number of patients who were prescribed CAM monotherapy for 7 days or for 90 days or longer. This study defined CAM monotherapy as prescription of no other drug but CAM, such as a generalized anti-bacterial drug (ATC code: J01) or an anti-mycobacterial drug (ATC code: J04), during the observation period. This study used PostgreSQL (Ver. 9.6) to construct the database and process the data and used R (Ver. 3.4.2) for statistical analyses.

In this analysis, the insurance claims data of the JMDC we used this time was the data unsuitable for analysis because information on patients was distributed in multiple tables and stored. In the case where it is necessary to consider the context of the time series such as dates peculiar to the cohort study conducted this time, engineering measures were necessary.

In this study, the schema (time series schema) storing time series information such as dates was created separately. Patients were extracted by specifying date information described in the time series schema in SQL extraction condition (where clause) when extracting patients. For example, the above method was used for processing to extract a patient prescribed CAM after the diagnosis confirmation date of MAC Lung disease. Since the data used this 
time is long-term insurance claims data for 12 years, it is considered that it was practically difficult without this method.

\section{RESULTS}

The patient data extracted using the eligibility criteria established for this study resulted in identification of 1211 insured individuals who met the eligibility criteria and 105 individuals who were excluded according to the exclusion criteria, leaving 1107 individuals as our analysis subjects. These individuals are referred to as patients with MAC Lung Disease. Table 1 shows the number of patients and rate of incidence categorized by age and gender as compared to the population and also shows results of statistical analyses for patients with MAC Lung Disease. Table 2 shows medication prescription conditions of the patients with MAC Lung Disease. Sixteen individuals $(1.4 \%)$ were prescribed CAM monotherapy for 7 days and 114 individuals (10.3\%) were prescribed CAM monotherapy for 90 days or longer. Among the 114 subjects, the prescription amount of clarithromycin was $200 \mathrm{mg}$ per day at the minimum and $1200 \mathrm{mg}$ per day at the maximum (Tables 1 and 2).

\section{DISCUSSION}

Rate of incidence of MAC Lung Disease increased with age and women accounted for $65 \%$ of included subjects, as shown in Table 1. Although conclusions cannot be drawn for subjects over 75 years of age, these results agree with the epidemiological statistic that most patients with MAC Lung Disease are middle-aged or elderly women.

A report speculates that CAM monotherapy may be done for patients who experience adverse drug reactions and hate longterm treatment with multiple agents [11]. Reports suggest that MAC bacteria acquire drug resistance to CAM fairly easily in trial environments [12]. As MAC Lung Disease does not resolve spontaneously, development of drug-resistant bacterial strains can be very problematic, particularly if bacteria also develop resistance to other treatments. Reports suggest that some MAC bacteria acquire genes from external bacterial species and mutate accordingly [13]. Therefore, mutation of MAC bacteria into a drug-resistant strain is a possibility. Although M. abscessus, a type of NTM, is rare, it could develop during treatment of patients with MAC Lung Disease [14]. Even in this case, it has been reported that CAM monotherapy may produce drug-resistant strains against macrolides [15]. As such, fundamentally, drug-resistance resulting from CAM monotherapy should be avoided [4].

After reviewing these cases, we believe it is imperative to investigate methods to disseminate suitable evidence to clinical practices, such as carefully reading package inserts of medications, following medical guidelines and heeding opinions of specialists that conform to these guidelines, in order to prevent cases were medication is prescribed without rational reasoning.

\section{CONSTRAINT}

We will describe constraints of this study. Firstly, the patient data used in this study included patients with illnesses that were identified and medications prescribed after onset of the observational period. This resulted in cases where the drug prescription conditions were potentially evaluated for a limited duration. However, since this study used data over a long period of time, the proportion of such patients is considered to be small.

\section{CONCLUSION}

This study clarified the rate of incidence of MAC Lung Disease and the ratio of cases that resulted in prescription of CAM monotherapy over a long duration by analyzing information in claims made by patients insured through society-managed health insurance plans. The results demonstrated that a number of these types of cases exist, even in clinical practice. However, since the drug administration duration for treatment of MAC Lung Disease is long, there is a possibility that administering certain drugs can be

Table 1: MAC Lung Disease: Number of patients.

\begin{tabular}{|c|c|c|c|c|c|c|c|}
\hline & & \multicolumn{2}{|c|}{ Population } & \multicolumn{2}{|c|}{ Patient } & \multicolumn{2}{|c|}{ Morbidity } \\
\hline & & \multicolumn{2}{|c|}{$(\mathrm{N}=5,478,478)$} & \multicolumn{2}{|c|}{$(\mathrm{N}=1,107)$} & \multicolumn{2}{|c|}{ (Patient/Population) } \\
\hline & & Male & Female & Male & Female & Male (\%) & Female (\%) \\
\hline \multirow{7}{*}{ Age (Years) } & $0-20$ & $8,40,068$ & $7,45,839$ & 7 & 4 & 0.00083 & 0.00054 \\
\hline & $21-30$ & $5,85,302$ & $4,26,339$ & 6 & 11 & 0.00273 & 0.00258 \\
\hline & $31-40$ & $5,07,761$ & $4,60,663$ & 36 & 43 & 0.00709 & 0.00933 \\
\hline & $41-50$ & $4,65,665$ & $4,24,423$ & 70 & 145 & 0.01503 & 0.03416 \\
\hline & $51-60$ & $3,34,462$ & $3,06,105$ & 103 & 245 & 0.0308 & 0.08004 \\
\hline & $61-70$ & $2,02,073$ & $1,45,434$ & 117 & 192 & 0.0579 & 0.13202 \\
\hline & $71-75$ & 13,634 & 20,710 & 30 & 38 & 0.22004 & 0.18349 \\
\hline Total & & $29,48,965$ & $25,29,513$ & 379 & 728 & 0.01285 & 0.02878 \\
\hline
\end{tabular}

Table 2: Drug prescription conditions for MAC Lung.

\begin{tabular}{|c|c|c|c|c|c|c|c|}
\hline & \multirow{3}{*}{$\begin{array}{c}\text { CAM } \\
\mathrm{N} \\
\end{array}$} & \multirow{2}{*}{\multicolumn{2}{|c|}{$\begin{array}{c}\text { Single-agent CAM } \\
\text { (For } 7 \text { days) }\end{array}$}} & \multirow{2}{*}{\multicolumn{2}{|c|}{$\begin{array}{c}\text { Single-agent CAM } \\
\text { (8-89 days) }\end{array}$}} & \multirow{2}{*}{\multicolumn{2}{|c|}{$\frac{\text { Single-agent CAM }}{\text { (For } 90 \text { days or longer) }}$}} \\
\hline & & & & & & & \\
\hline & & $\mathrm{N}$ & $\%$ & $\mathrm{~N}$ & $\%$ & $\mathrm{~N}$ & $\%$ \\
\hline Male & 379 & 7 & 1.8 & 90 & 23.7 & 31 & 8.2 \\
\hline Female & 728 & 9 & 1.2 & 103 & 14.1 & 83 & 11.4 \\
\hline Total & 1107 & 16 & 1.4 & 193 & 17.4 & 114 & 10.3 \\
\hline
\end{tabular}


detrimental to patient health if not done appropriately. Through our research, we want to raise awareness of the importance of prescribing medications appropriately and based on suitable evidence in clinical practice. In the immediate future, it seems to be effective to develop an educational system on single agent administration of antimicrobial agents. In the future, it is desirable to establish a mechanism by which a dangerous single agent administration can be prevented beforehand by a computer system using AI technology or the like.

\section{ACKNOWLEDGEMENT}

This study was executed with the help of a research grant from the Japan Society for the Promotion of Science (Grant number is $17 \mathrm{~K} 17816)$ and I thank Ms. Shigeko Noguchi who gave me the opportunity to conduct this research.

\section{ETHICAL APPROVAL}

As the insurance claim data was anonymized data without intervention in a completely anonymized form, approval from an ethics committee was not necessary according to the rules of the affiliated institution.

\section{CONFLICT OF INTEREST}

The author declares no conflict of interest.

\section{REFERENCES}

1. Yon Ju R, Won-Jung K, Daley CL. Diagnosis and treatment of nontuberculous mycobacterial lung disease: clinicians' perspectives. Tuberc Respir Dis. 2016;79(2):74-78.

2. Namkoong H, Kurashima A, Morimoto K, Hoshino Y, Hasegawa N, Ato $\mathrm{M}$, et al. Epidemiology of pulmonary nontuberculous mycobacterial disease, Japan. Emerg Infect Dis. 2016;22(6):1116-1117.

3. The Nontuberculous Mycobacteriosis control committee of the Japanese society for tuberculosis. The scientific assembly for infection and tuberculosis of the japanese respiratory society. Guidelines for chemotherapy of pulmonary nontuberculous mycobacterial disease-2012 Revised Version. Kekkaku. 2013;88(1):29-32.

4. Griffith DE, Aksamit T, Brown-Elliott BA, Catanzaro A, Daley C, Gordin F, et al. An official ats/idsa statement: Diagnosis, treatment and prevention of nontuberculous mycobacterial diseases. American
Journal of Respiratory and Critical Care Medicine. 2007;175(4):367416.

5. Haworth CS, Banks J, Capstick T, Fisher AJ, Gorsuch T, Laurenson IF, et al. British thoracic society guidelines for the management of non-tuberculous mycobacterial pulmonary disease (NTM-PD). Thorax. 2017;72(2):ii1-ii64.

6. Kobashi Y, Matsushima T. The microbiological and clinical effects of combined therapy according to guidelines on the treatment of pulmonary Mycobacterium avium complex disease in Japan-including a follow-up study. Respiration. 2007;74(4):394-400.

7. David S. Tuberculosis and Nontuberculous mycobacterial infections, Seventh edition, ASM Press. 2017.

8. Ichikia H, Watanabeb A, Uedab S, Satob C, Abeb M. Clinical course and imaging findings of factors exacerbating pulmonary Mycobacterium avium complex disease. The Journal of the Japanese Respiratory Society (Nihon Kokyuki Gakkai Zasshi). 2014;3:783-788.

9. Kwon YS, Koh WJ. Diagnosis and treatment of nontuberculous mycobacterial lung disease. J Korean Med Sci. 2015;31(5):649-659.

10. Japan Medical Data Center. JMDC Claims Database. 2018.

11. Katsuhiro K, Toshiaki T. Clinical features and treatment history of clarithromycin resistance in $\mathrm{M}$. avium-intracellulare complex pulmonary disease patients. The Journal of the Japanese Respiratory Society (Nihon Kokyuki Gakkai Zasshi). 2007;45:587-592.

12. Meier A, Kirschner P, Springer B, Steingrube VA, Brown BA, Wallace RJ, et al. Identification of mutations in 23S rRNA gene of Clarithromycin-resistant Mycobacterium intracellulare. Antimicrob Agents Chemother. 1994;38(2):381-384.

13. Yano H, Iwamoto T, Nishiuchi Y, Nakajima C, Starkova DA, et al. Population structure and local adaptation of MAC lung disease agent Mycobacterium avium subsp. hominissuis. Genome Biol Evol. 2017;9(9):2403-2417.

14. Tsunoda Y, Sekine A, Sumazaki Y, Tanaka T, Lin SY, Takoi H, et al. Mycobacterium abscessus pulmonary disease: Important pathogen involved in microbial substitution during the treatment of nonabscessus mycobacterial disease. Kekkaku. 2016;91(4):469-473.

15. Lee MR, Sheng WH, Hung CC, Yu CJ, Lee LN, Hsueh PR. Mycobacterium abscessus complex infections in humans. Emerg Infect Dis. 2015;21(9):1638-1646. 